\title{
Concept of Mobile Measuring Station as an Element of Smart Cities of the Future
}

\author{
Weronika Janc $^{1}$, Krzysztof Sitko ${ }^{1}$, Kamil Kopeć ${ }^{1}$, Grzegorz Sierpiński ${ }^{*}$ \\ ${ }^{1}$ Silesian University of Technology, Faculty of Transport and Aviation Engineering, Department of Transport Systems and \\ Traffic Engineering \\ Krasińskiego Str. 8, 40-019 Katowice, POLAND \\ * grzegorz.sierpinski@polsl.pl
}

\section{Extended Abstract}

Knowledge of the traffic volume value is necessary to determine the distribution of traffic on the road network, his tendency to change, for example, in individual hours or days. By obtaining such data, it is possible to prepare forecasts of the load on roads and to determine Level of Service. The juxtapostion of the obtained traffic intensity values allows the determination of peak hours and hours of occasional traffic, which in turn allows to determine the area in which changes the organization of traffic could or should arise. The results of traffic volume examination are also used to make a decision about road classification, determination of road course and prioritising them in a road network. This is extremely important, especially now that streets and intersections in cities are becoming more and more congested.

The research project involved the construction and preparation of a mobile measuring station. Application at this station a specialized license plate recognition camera allows to obtain data on the number of passing vehicles over time on the road section under investigation, together with information on which lane the vehicle was moving. More these stations on one intersection can be used to receive other traffic parameters, (like a directional structure). It means extension of stations can contribute to improvement in determination of traffic parameters, including torsion relations on the crossroads.

Next step of the research project assumed different measurement scenarios to test prepared station. The following assumptions were made: different lighting conditions, different weather conditions and different traffic intensity at one of the junction inlets. The analysis of the measurements relate to the recording from the camera memory and their conformity with reality with regard to the number of vehicles and the lane on which the vehicle is moving. Thanks to the method of manual measurements simultaneously performed by observers, it is possible to indicate the limitations associated with the use of the proposed device.

\section{Acknowledgements}

The present research has been financed within the framework of the $1^{\text {st }}$ competition for funding student's research projects ("Research University - Excellence Initiative") as part of the project: "Determining the possibility of using vehicle detection in a mobile measurement device as an element of smart cities of the future". 